\title{
STABILITY ANALYSIS OF A MESHLESS METHOD IN IRREGULAR NODAL DISTRIBUTIONS FOR FLOW PROBLEMS
}

\author{
G. KOSEC \\ Jožef Stefan Institute, Department of Communication Systems, Jamova 391000 Ljubljana, Slovenia
}

\begin{abstract}
This paper is focused on the analysis of the numerical solution of flow problems in irregular domains. The numerical approach is based on the weighted least squares (WLS) approximation constructed over the local support domain, i.e. a sub cluster of computational nodes, to evaluate partial differential operators, in our case spatial derivatives up to second order. There are several possibilities for elegant formulation as well as computer implementation of such method, which are first and foremost consequence of the fact that the node has to be aware only of the distance to other nodes, i.e. no topological relation between nodes is required. The presented meshless approach is applied on the lid-driven cavity problem in randomly generated domain. It is demonstrated that using adequately wide support domains, i.e. enough support nodes with a proper weighting, provide stable results even in highly deformed domains, however, at the cost of the accuracy and computational complexity, especially in cases when the support domain changes during the computation. The optimal meshless configuration, i.e. support of 15 nodes weighted with Gaussian weight function and monomials up to second order as basis, is suggested based on experimental analyses. The results are presented in terms of comparison with already published data on regular nodal distributions, convergence analysis on regular nodal distribution and stability analysis of the solution with respect to the level of nodal irregularity and local support size. Keywords: fluid flow, meshless, parallel, stability.
\end{abstract}

\section{INTRODUCTION}

The core of flow problems is the solution of Navier-Stokes Equation [1] or its variants, e.g. Darcy or Brinkman equation for flow in porous media. Since the problem is usually not solvable in a closed form numerical methods such as the Finite Volume Method (FVM), Finite Difference Method (FDM), or the Finite Element Method (FEM) are employed to numerically tackle the problem. Besides mesh based methods a class of so-called meshless methods that are based on scattered discretization nodes [2] can be used. In this paper, one of the simplest class of MMs, Meshless Local Strong Form Method (MLSM), a generalization of methods, which are in literature also known as Diffuse Approximate Method (DAM) [3], Local Radial Basis Function Collocation Methods (LRBFCM), Generalized FDM [4], Collocated discrete least squares (CDLS) meshless [5], etc., is used. The main difference between mesh based and meshless methods is that meshless methods shift the determination of relation between nodes into the solution procedure. The final goal of such an approach is higher flexibility in complex domains, moving boundaries and nodal adaptivity.

Although the meshless methods do not require any predefined relations between nodes and even randomly distributed nodes could be used, it is well-known that using regularly distributed nodes lead to more accurate and more stable results [6-8], which is also confirmed in this paper. Therefore, despite meshless seeming robustness regarding the nodal distribution, a certain effort has to be invested into the positioning of the nodes. This paper deals with the analysis of MLSM in randomized nodal distributions. 


\section{METHODOLOGY}

The core of the spatial discretization used in this paper is a local approximation of a considered field over the overlapping local support domains, i.e. in each node we use approximation over a small local sub-set of neighbouring $n$ nodes. The trial function is thus introduced as

$$
\hat{u}(p)=\sum_{i}^{m} a_{i} b_{i}(\mathbf{p})=\mathbf{b}(\mathbf{p})^{T} a,
$$

With $m, \alpha, \mathbf{b}, \mathbf{p}\left(p_{x}, p_{y}\right)$ standing for the number of basis functions, approximation coefficients, basis functions and the position vector, respectively. When the number of basis functions and number of support domain is the same $n=m$ the determination of coefficients simplifies to solving a system of $n$ linear equations that results from expressing eqn (1) in each support node.

$$
u\left(\mathbf{p}_{j}\right)=\mathbf{u}=\mathbf{b}(\mathbf{p})^{T} \alpha,
$$

$\mathbf{p}_{i}$ are positions of support nodes and $\mathbf{u}$ are values of considered field in the support positions. The above system can be written in a matrix form as

$$
\mathbf{u}=\mathbf{B} \boldsymbol{\alpha},
$$

where $\mathbf{B}$ stands for coefficient matrix with elements $\mathrm{B}_{j i}=b_{i}\left(\mathbf{p}_{j}\right)$. System (3) can be effectively solved with Cholesky decomposition. The most known method that use such an approach is LRBFCM that has been recently used in various problems $[9,10]$. If the number of support nodes is higher than the number of basis functions $n>m$ a Weighted Least Squares (WLS) approximation is used to solve over-determined problem. An example of such approach is DAM [3] that was originally formulated to solve fluid flow in porous media. DAM uses six monomials for basis and nine nodded support domains to evaluate first and second derivatives of physical fields required to solve problem at hand. To determine the approximation coefficients, a norm

$$
r^{2}=\sum_{j}^{n} W\left(\mathbf{p}_{j}\right)\left(u\left(\mathbf{p}_{j}\right)-\hat{u}\left(\mathbf{p}_{j}\right)\right)^{2}=(\mathbf{B} \alpha-\mathbf{u})^{T} \mathbf{W}(\mathbf{B} \alpha-\mathbf{u}),
$$

is minimized, where $\mathbf{W}$ is a diagonal matrix with elements $W_{j j}=W\left(\mathbf{p}_{j}\right)$ with

$$
W(\mathbf{p})=\exp \left(-\left(\frac{\left\|\mathbf{p}_{0}-\mathbf{p}\right\|}{\sigma p_{\min }}\right)^{2}\right)
$$

where $\sigma$ stands for weight parameter, $\mathbf{p}_{0}$ for centre of support domain and $p_{\min }$ for the distance to the first support domain node. There are different approaches to solve (4). The most intuitive and also computationally effective is to simply compute gradient of (4) with respect to the resulting in

$$
\mathbf{W B B}^{\mathrm{T}} \boldsymbol{\alpha}=\mathbf{W B}^{\mathrm{T}} \mathbf{u}
$$

The problem of above approach is bad conditioning. More stable and more expensive approach is QR decomposition. Even more stable is SVD decomposition, which is off course also more expensive. Nevertheless, the problem can be written in matrix form as

$$
a=\left(\mathbf{W}^{0.5} \mathbf{B}\right)^{+} \mathbf{W}^{0.5} \mathbf{u}
$$


where $\left(\mathbf{W}^{0.5} \mathbf{B}\right)^{+}$stand for a Moore-Penrose pseudo inverse. By explicit expression of $\alpha$ into the (2) an equation

$$
\hat{u}(\mathbf{p})=\mathbf{b}(\mathbf{p})^{T}\left(\mathbf{W}^{0.5}(\mathbf{p}) \mathbf{B}\right)^{+} \mathbf{W}^{0.5}(\mathbf{p}) \mathbf{u}=\chi(\mathbf{p}) \mathbf{u},
$$

is obtained, where $\chi$ stand for the shape functions. Now, we can apply partial differential operator, which is our goal, on the trial function,

$$
L \hat{u}(\mathbf{p})=L \chi(\mathbf{p}) \mathbf{u},
$$

where $L$ stands for general differential operator. In this paper we deal with a Navier-Stokes equation and therefore only shape functions for Laplace operator and first derivatives are needed, which are pre-computed and stored

$$
\begin{aligned}
& \chi^{\partial x}(\mathbf{p})=\frac{\partial}{\partial x} \mathbf{b}(\mathbf{p})^{T}\left(\mathbf{W}^{0.5}(\mathbf{p}) \mathbf{B}\right)^{+} \mathbf{W}^{0.5} \\
& \chi^{\partial y}(\mathbf{p})=\frac{\partial}{\partial x} \mathbf{b}(\mathbf{p})^{T}\left(\mathbf{W}^{0.5}(\mathbf{p}) \mathbf{B}\right)^{+} \mathbf{W}^{0.5} \\
& \chi^{\Delta^{2}}(\mathbf{p})=\frac{\partial}{\partial x} \mathbf{b}(\mathbf{p})^{T}\left(\mathbf{W}^{0.5}(\mathbf{p}) \mathbf{B}\right)^{+} \mathbf{W}^{0.5}
\end{aligned}
$$

Although the selection of basis function $\mathbf{b}$ is general, several researchers follow the results from Franke's analysis [11] and use Hardy's Multiquadrics; however, in this work the monomials up to second order $\left(1, p_{x}, p_{y}, p_{x}^{2}, p_{y}^{2}, p_{x}, p_{y}\right)$ are used based on the results presented in [12].

The presented formulation is convenient for implementation since most of the complex operations, i.e. finding support nodes and building shape functions, are performed only when nodal topology changes. In the main simulation, the pre-computed shape functions are then convoluted with the vector of field values in the support to evaluate the desired operator.

The presented approach is even easier to handle than the FDM; however, despite its simplicity it offers many possibilities for treating challenging cases, e.g. nodal adaptivity to address regions with sharp discontinuities or p-adaptivity to treat obscure anomalies in physical field, furthermore, the stability versus computation complexity and accuracy can be regulated simply by changing number of support nodes, etc. All these features can be controlled on the fly during the simulation by re computing the shape functions with different setups. However, such re-setup is expensive, since the $\mathbf{b b}(\mathbf{p})^{\mathrm{T}}\left(\mathbf{W}^{0.5}(\mathbf{p}) \mathbf{B}\right)^{+} \mathbf{W}^{0.5}$ has to be re-evaluated, with asymptotical complexity of $O\left(N_{D} n m^{2}\right)$, where $N_{D}$ stands for total number of discretization nodes. In addition, the determination of support domain nodes also consumes some time, for example, if a $\mathrm{kD}$-tree [13] data structure is used, first the tree is built with $O\left(N_{D}\left(\log N_{D}\right)\right.$ and then additional $O\left(N_{D}\left(\log N_{D}+n\right)\right)$ for collecting $n$ supporting nodes.

\section{RESULTS}

The presented MLSM is tested on a lid-driven cavity problem that has been proposed in 1982 [14] and it is still widely studied for validation of novel methods and numerical principles, for example, recently for meshless methods [15-17]. The problem is defined as

$$
\nabla \cdot \mathrm{v}=0
$$




$$
\frac{\partial \mathrm{v}}{\partial t}+\nabla \cdot(\mathrm{vv})=-\nabla P+\frac{1}{\operatorname{Re}} \nabla^{2} \mathrm{v}
$$

where $\mathbf{v}, t, P$, and Re stand for dimensionless velocity, time, pressure, and Reynolds number, respectively. Zero velocity boundaries are assumed except the lid velocity that is set to 1 . Initial pressure and velocity are set to zero. The problem is solved with MLSM spatial discretization, explicit two level temporal stepping and artificial compressibility method for pressure treatment [18].

First, we want to be sure that the MLSM provides accurate results on regular nodal distributions. This is tested by comparison of MLSM results at three different numbers, namely $\mathrm{Re}=[100,1000,3200]$, against three different solutions, namely, Mramor [15], Sahin [19] and Ghia [20]. In Fig. 1 a spatial convergence in terms of maximal $v_{y}$ on a range from $N=121$ $(11 \times 11)$ to $N=315844(562 \times 562)$ uniformly distributed nodes is presented, together with a reference data. It can be seen that for all cases results converge towards reference solutions. Note that the reference solutions do not represent the convergence behaviour and are added only for the sake of comparison.

In this paper we are more interested on the impact of support domain deformation on the computation efficiency. To test this behaviour, a randomized nodal distribution is introduced, i.e. the initially uniformly distributed nodes are translated by random offsets

$$
\mathbf{p}_{i} \leftarrow \mathbf{p}_{i}+D \mathbf{r} \Delta p
$$

where $\mathbf{r}=\left(r_{x}, r_{y}\right)$ is vector of random numbers within $[-1,1], D$ is deformation magnitude, $\mathbf{p}_{i}$ is position of $i$-th node, and $\Delta p$ is spatial step of original uniform nodal distribution. In Fig. 2 the maximal horizontal cross-section velocity with respect to deformation magnitude and number of support nodes for different weight parameters is presented.

The very first thing we see is that by increasing the deformation the results becomes unstable, especially; when the support is small, i.e. small number of nodes influences the

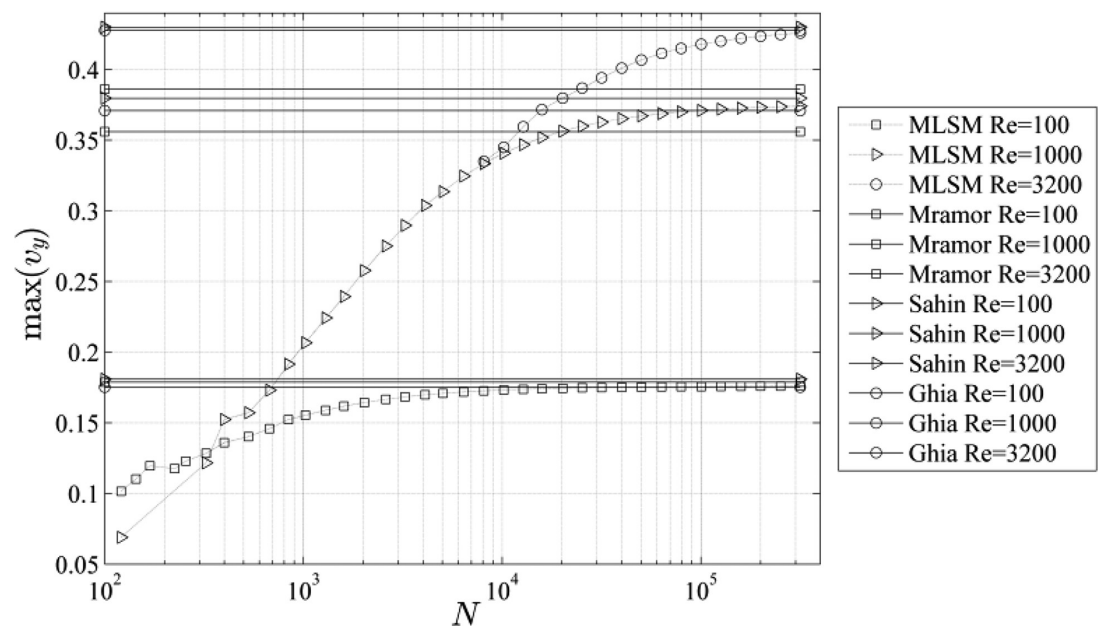

Figure 1: The maximal mid-plane velocity $\max \left(v_{y}\left(p_{x}, 0.5\right)\right)$ with respect to the number of computational nodes for different Re numbers. Horizontal lines stand for reference solutions. 

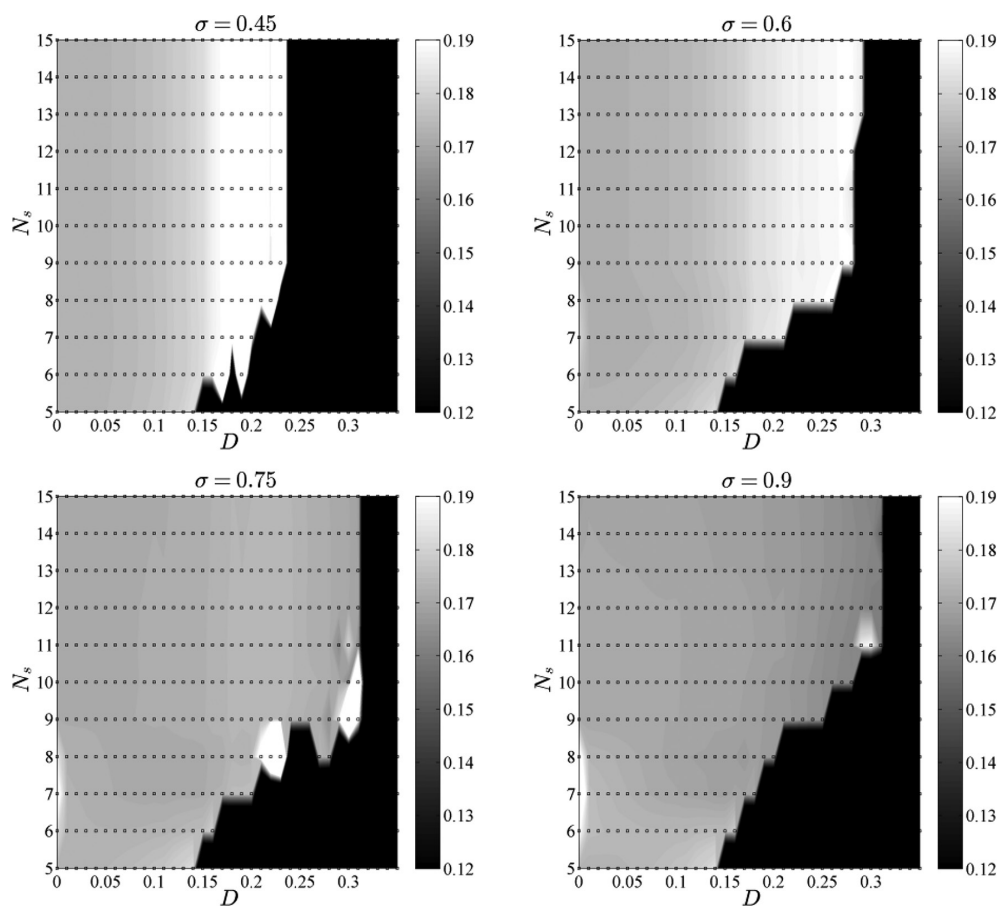

Figure 2: The maximal horizontal cross-section velocity with respect to deformation magnitude and number of support nodes for different weight parameters.

approximation function, as a consequence of a small number of support nodes and/or the small weight parameter. However, increasing the weight parameter as well as number of support nodes, improves results. Figure 2 serves mainly to determine the stability limit for given setup, where the instable setups are represented with zero values, shown as black areas on the figure. More precise representation of this behaviour is presented in Fig. 3, where it can be also seen that the wider supports result in a lower velocities due to the fact that WLS approximation with wide support domains fails to capture all the details in the field. It is important to note that the number of support nodes also influences the computational time since the generation of shape functions can be estimated to complexity $O(\mathrm{~nm})^{2}$ and the evaluation of partial operators to $O(n)$.

From above results we select 15 support nodes weighted with $\sigma=0.75$ as reasonable tradeoff between stability, accuracy and computation cost that provides stable results. Finally, in Fig. 4, examples of proposed MLSM solution of lid-driven problem on regular and nonregular domains are presented.

The introduced formulation has many beneficial effects. One of the most attractive is the generality. Note that equations presented in section 2 do not require explicit knowledge about dimensionality of the domain. Also the approximation type, basis size, number of support nodes are all free parameters. Such generality can be directly implemented in generic languages like $\mathrm{C}++$. Results presented in this paper are all computed with a such generic meshless code that can be downloaded from reference [21]. Besides simplicity and straightforward implementation, there are many opportunities to fully exploit modern computer architectures through different parallel computing strategies [6]. 


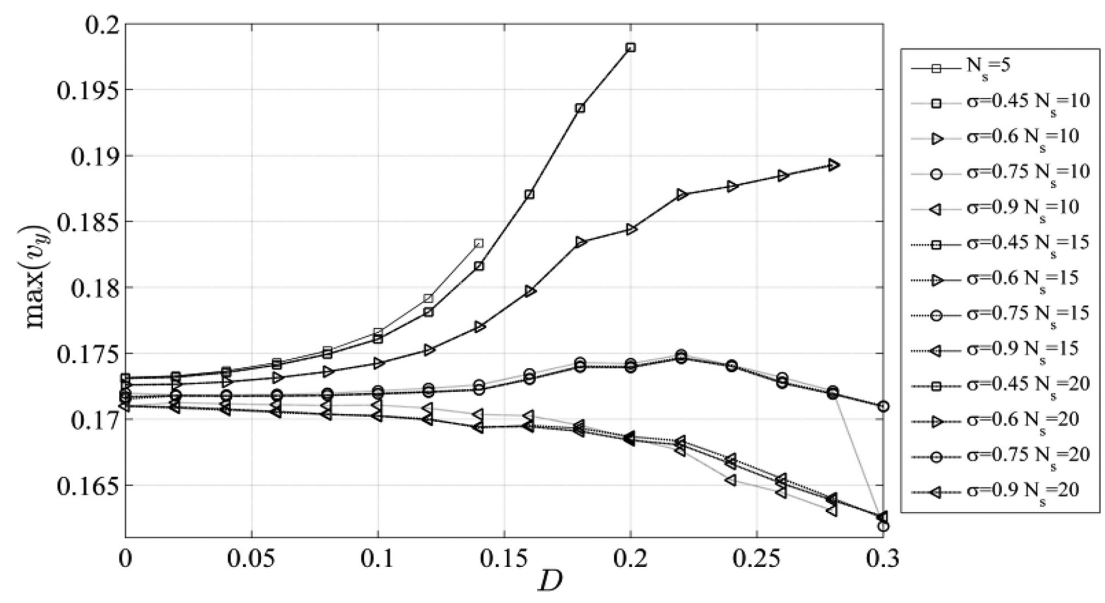

Figure 3: The maximal horizontal cross-section velocity as a function of deformation magnitude, number of support nodes and weight parameter.
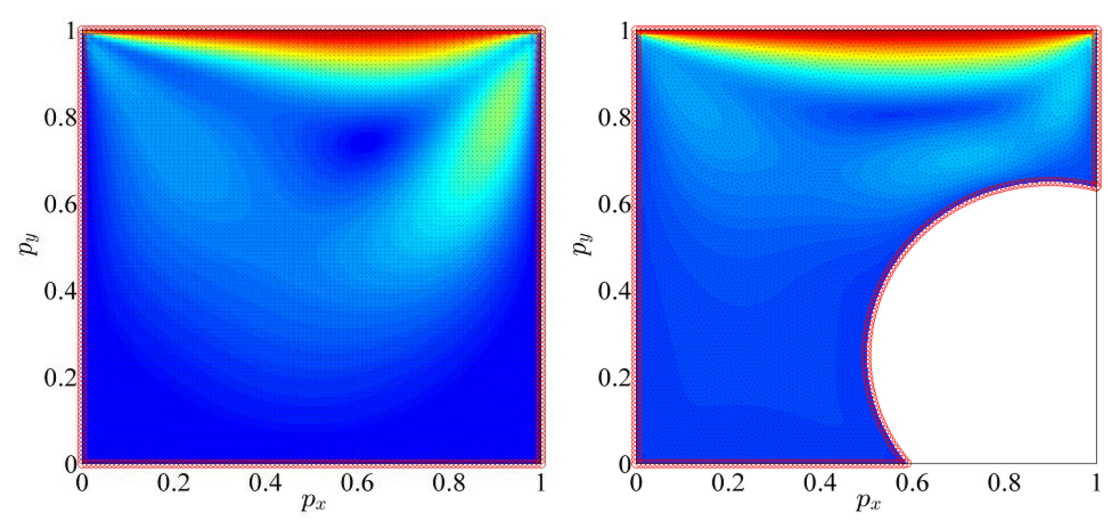

Figure 4: The velocity magnitude contour plot of lid-driven cavity solution in two different domains.

\section{CONCLUSIONS}

This paper deals with a MLSM solution of a Navier-Stokes equation on randomized nodal distributions, with the goal to identify the most suitable MLSM setup for computations of flow problems on irregular domains. First, it is demonstrated, by comparison against already published data and convergence analysis, that the MLSM performs well on regular nodal distributions. In next step the nodes are scattered by random offsets to mimic the consideration of more complex domains [22]. As expected, the randomization destabilized the solution; however, increasing the support domain improves the results with respect to the nodal deformation. However, such stabilization comes with a price in accuracy and computational complexity. Ultimately, a setup with $\sigma=0.75$ and support size of 15 nodes is chosen as a reasonable tradeoff between stability, accuracy and computation cost that provides stable results.

Future work will be focused on implementation of more complex physical models, more detailed analysis of MLSM application on complex 3D domains. 


\section{ACKNOWLEDGMENT}

We acknowledge the financial support from the Slovenian Research Agency under the program group P2-0095.

\section{REFERENCES}

[1] Ferziger, J.H. \& Perić, M., Computational Methods for Fluid Dynamics, Springer: Berlin, 2002. http://dx.doi.org/10.1007/978-3-642-56026-2

[2] Li, S. \& Liu, K.W., Meshfree and particle methods and their applications. Applied Mechanics Reviews, 55, pp. 1-34, 2002. http://dx.doi.org/10.1115/1.1431547

[3] Wang, C.A., Sadat, H. \& Prax, C., A new meshless approach for three dimensional fluid flow and related heat transfer problems. Computers and Fluids, 69, pp. 136-146, 2012. http://dx.doi.org/10.1016/j.compfluid.2012.08.017

[4] Yang, C., Tang, D., Yuan, C., Kerwin, W., Liu, F., Canton, G., Hatsukami, T.S. \& Atluri, S., Meshless generalized finite difference method and human carotid atherosclerotic plaque progression simulation using multi-year MRI patient-tracking data. CMES: Computer Modeling in Engineering \& Sciences, 28, pp. 95-107, 2008.

[5] Arzani, H. \& Afshar, M.H., Solving Poisson's equation by the discrete least square meshless method. WIT Transactions on Modelling and Simulation 42, pp. 23-31, 2006. http://dx.doi.org/10.2495/be06003

[6] Trobec, R \& Kosec, G., Parallel Scientific Computing: Theory, Algorithms, and Applications of Mesh Based and Meshless Methods, Springer, 2015. http://dx.doi.org/10.1007/978-3-319-17073-2

[7] Trobec, R., Kosec, G., Šterk, M. \& Šarler, B., Comparison of local weak and strong form meshless methods for 2-D diffusion equation. Engineering Analysis with Boundary Elements, 36, pp. 310-321, 2012. http://dx.doi.org/10.1016/j.enganabound.2011.08.009

[8] Amani, J., Afshar, M.H. \& Naisipour, M., Mixed discrete least squares meshless method for planar elasticity problems using regular and irregular nodal distributions. Engineering Analysis with Boundary Elements, 36, pp. 894-902, 2012. http://dx.doi.org/10.1016/j.enganabound.2011.09.012

[9] Kosec, G. \& Trobec, R, Simulation of semiconductor devices with a local numerical approach. Engineering Analysis with Boundary Elements, 50, pp. 69-75, 2015. http://dx.doi.org/10.1016/j.enganabound.2014.07.013

[10] Kosec, G. \& Šarler, B., Simulation of macrosegregation with mesosegregates in binary metallic casts by a meshless method. Engineering Analysis with Boundary Elements, 45, pp. 36-44, 2014. http://dx.doi.org/10.1016/j.enganabound.2014.01.016

[11] Franke, J., Scattered data interpolation: tests of some methods. Mathematics of Computation, 48, pp. 181-200, 1982.

[12] Kosec, G. \& Zinterhof, P., Local strong form meshless method on multiple Graphics Processing Units. CMES: Computer Modeling in Engineering \& Sciences, 91, pp. 377396, 2013.

[13] Zachmann, G. \& Langetepe, E., Geometric Data Structures for Computer Graphics, A. K. Peters Ltd, Wellesley: MA, 2006. 
[14] Ghia, U., Ghia, K.N. \& Shin, C.T., High-Re solutions for incompressible flow using the Navier-Stokes equations and a multigrid method. Journal of Computational Physics $\mathbf{4 8 ,}$ pp. 387-411, 1982.

http://dx.doi.org/10.1016/0021-9991(82)90058-4

[15] Mramor, K., Vertnik, R. \& Šarler, B., Low and intermediate re solution of lid driven cavity problem by local radial basis function collocation method. CMC: Computers, Materials, \& Continua, 1, pp. 1-21, 2013.

[16] Bustamante, C.A., Power, H., Sua, Y.H. \& Florez, W.F., A global meshless collocation particular solution method (integrated Radial Basis Function) for two-dimensional Stokes flow problems. Applied Mathematical Modelling, 37, pp. 4538-4547, 2013. http://dx.doi.org/10.1016/j.apm.2012.09.069

[17] Bourantas, G.C. \& Loukopoulos, V.C., A meshless scheme for incompressible fluid flow using a velocity-pressure correction method. Computers \& Fluids, 88, pp. 189199, 2013.

http://dx.doi.org/10.1016/j.compfluid.2013.09.010

[18] Arpino, F., Massarotti, N., Mauro, A. \& Nithiarasu, P., Artificial compressibility based CBS solutions for double diffusive natural convection in cavities. International Journal of Numerical Methods for Heat \& Fluid Flow, 23, pp. 205-225, 2013. http://dx.doi.org/10.1108/09615531311289196

[19] Sahin, M. \& Owens, R., A novel fully implicit finite volume method applied to the liddriven cavity problem. PartI: High Reynolds number flow calculations. International Journal for Numerical Methods in Fluids, 42, pp. 57-77, 2003. http://dx.doi.org/10.1002/fld.442

[20] Ghia, U., Ghia, K. \& Shin, C., High-Re solutions for incompressible flow using the Navier-Stokes equations and a multigrid method. Journal of Computaional Physics, $\mathbf{4 8 ,}$ pp. 387-411, 1982.

http://dx.doi.org/10.1016/0021-9991(82)90058-4

[21] Kosec, G., Kolman, M. \& Slak, J., http://www-e6.ijs.si/ParallelAndDistributedSystems/PDE_solver_utils/html/index.html, 2016.

[22] Kosec, G., Meshless Solution of Incompressible Flows in Complex Domains, Proceedings of the Ninth International Conference on Engineering Computational Technology, Civil-Comp Press, 2014. 